\author{
Stefan Odenbach
}

\title{
Magnetic hybrid materials
}

Received: 12 December 2018 / Accepted: 14 December 2018 / Published online: 27 December 2018

(C) Springer-Verlag GmbH Germany, part of Springer Nature 2019

Magnetic hybrid materials, i.e., magnetic nano- or microparticles embedded in a nonmagnetic matrix, have attracted scientific interest since more than 50 years. The main reason for this long-lasting scientific activity is given by the fact that these materials allow a change of their properties and mechanical behavior, for example of their flow, if a liquid matrix is used, due to the action of external magnetic fields.

Since magnetic fields can easily be controlled concerning their strength and spatial distribution, tailored forces can be exerted to magnetic hybrid materials giving rise to the development of applications as well as new opportunities for basic research.

Looking on the complete class of magnetic hybrid materials, one has to distinguish between those having a liquid matrix and those with an elastic one.

In case that the matrix is liquid, one has to distinguish which kind of particles is used. If magnetic nanoparticles are used, one obtains a so called ferrofluid which represents a long-term stable suspension stabilized by Brownian motion of the particles. In these fluids, flow control by magnetic fields can be exhibited, changes of the viscosity occur, and numerous applications from technical concept like sealing of rotating shafts to medical treatment, e.g., in cancer therapy have been investigated and established. Using magnetic microparticles in so called magnetorheological fluids (MR-fluids), one obtains significantly higher changes of viscosity in the presence of magnetic fields, which are induced by formation of particle chains. These changes can be used to design dampers or clutches which are meanwhile commercialized. Nonetheless, MR-fluids have always the problem that microparticles will sediment.

Within this special issue, we will focus on those magnetic hybrid materials having an elastic matrix. Obviously, magnetic microparticles can here be used without sedimentation problems as long as the particles are homogeneously distributed during the polymerization process.

In these magnetorheological elastomers or gels, changes of the E-modulus of the material can be induced by the action of magnetic fields. This class of magnetic hybrid materials, which finds scientific interest since about 10 years only, is actually growing dramatically. Changes of the matrix from elastomers to gels and hydrogels, the use of nano- and microparticles as well as the choice of magnetically hard and magnetically soft particles or mixtures of them, have shown that a tremendous range of properties and possibilities for magnetic influence can be created by a sufficient choice of the composition of the magnetorheological elastomers.

Focusing on potential technical applications of such magnetic hybrid materials, it is quite obvious that a tailored specification of their field-dependent properties is required. Due to the highly complex interaction between the particles, the particles and the magnetic field and the particles and their elastic surrounding an $a$ priori description or a description using simplified standard models will not lead to sufficient results. A real understanding of these materials can only be achieved on a scale bridging basis starting with a microscopic description of particle-particle and particle-matrix interaction and ranging toward meso- and macroscopic material models based on microscopic material behavior. This scale bridging approach became possible during 
the past years as well due to new development in theoretical description as due to new experimental techniques allowing to combine microscopic changes with macroscopic alterations of the material properties.

It is the central goal of the DFG priority program 1681 "Field-controlled particle matrix interactions: synthesis, multiscale modeling and application of magnetic hybrid materials" to promote such a scale bridging comprehension of magnetic hybrid materials. Based on the work of this priority program, the Special Issue covers the whole range of fields of interest in this topic-from experimental techniques for macro- and microscopic characterization and theoretical modeling on various scales toward technical applications of such smart magnetic materials.

Publisher's Note Springer Nature remains neutral with regard to jurisdictional claims in published maps and institutional affiliations. 